\title{
Matter-Wave Emission in Optical Lattices: Single Particle and Collective Effects
}

\author{
Inés de Vega, ${ }^{*}$ Diego Porras, and J. Ignacio Cirac \\ Max-Planck-Institut für Quantenoptik, Hans-Kopfermann-Str. 1, Garching, D-85748, Germany
}

(Received 14 July 2008; published 31 December 2008)

\begin{abstract}
We introduce a simple setup corresponding to the matter-wave analogue of impurity atoms embedded in a photonic crystal and interacting with the radiation field. Atoms in a given internal level are trapped in an optical lattice, and play the role of the impurities. Atoms in an untrapped level play the role of the radiation field. The interaction is mediated by means of lasers that couple those levels. By tuning the lasers parameters, it is possible to drive the system through different regimes, and observe phenomena such as matter-wave superradiance, non-Markovian atom emission, and the appearance of bound atomic states.
\end{abstract}

DOI: 10.1103/PhysRevLett.101.260404

PACS numbers: 03.75.- b, 03.65.Yz, 42.50.-p

Recent progress in atomic physics has allowed experimentalists to trap atoms in optical potentials at very low temperatures. This has led to the observation of several interesting phenomena in which atom-atom interactions play a predominant role. With atoms loaded in optical lattices it is nowadays possible, e.g., to reach the strong correlation regime where quantum phase transitions between superfluid and insulator phases [1,2], TonksGirardeau gases [3], or even entanglement between neighboring atoms can be observed. Those experiments have triggered a large amount of theoretical work proposing and analyzing new experiments where intriguing condensed matter behavior could be observed [4].

In this work we show that with the same systems it is possible to observe a broad spectrum of phenomena usually connected to light-matter interactions (see [5-7] for related setups). Here, the role of matter is played by the absence or presence of one atom in the ground state of an optical potential, whereas the role of light is played by weakly interacting atoms in a different internal state which is not affected by the optical potential. The coupling between those two systems is induced by Raman lasers, which connect the two internal states of each atom (see Fig. 1). As we will show, the Hamiltonian that describes this situation is very similar to that describing the interaction between two-level atoms and the electromagnetic field within a photonic crystal (PC) [8] (see [9] for a tutorial of $\mathrm{PCs})$. By changing the laser and optical trapping parameters the system can be driven to different regimes where a rich variety of phenomena can be observed. These include the spontaneous symmetry breaking of the system predicted by the mean field theory [10], collective effects in the emission of atoms from the lattice [11,12], and the formation of a bound trapped-untrapped atom state. This state is analogous to the atom-photon bound state that appears when atoms embedded in a PC emit photons within the gap region, which remain exponentially localized around the atom and strongly coupled to it $[9,13,14]$. Moreover, it is possible to reach a regime in which weakly confined atoms drive atom-atom interactions between strongly confined ones, giving rise to effective Coulomblike interactions between them.

We consider $N$ cold atoms with a ground state hyperfine level $|a\rangle$ and frequency $\omega_{a}^{0}$ that is trapped by an optical lattice with $M$ sites and lattice period $d_{0}$. The atomic motion is restricted to the lowest Bloch band in the collisional blockade regime, where either one or no atom occupy each potential well of the lattice, which we will approximate by a harmonic oscillator of frequency $\omega_{0}$. Then, we can replace the creation operator at each site by $\sigma_{\mathbf{j}}^{+}=|1\rangle_{\mathbf{j}}\langle 0|$, which describes transitions from the Fock state $|0\rangle_{\mathbf{j}}$ with no atoms at site $\mathbf{j}$, to a state $|1\rangle_{\mathbf{j}}$ with one atom [5]. The atoms have an additional internal level, $|b\rangle$, that is not affected by the lattice potential and has a frequency $\omega_{b}$. We introduce the field operator $\psi_{b}^{\dagger}(\mathbf{r})=$ $(1 / \sqrt{V}) \sum_{\mathbf{k}} b_{\mathbf{k}}^{\dagger} e^{i \mathbf{k} \cdot \mathbf{r}}$, where $V$ is the quantization volume, and $b_{\mathbf{k}}^{\dagger}$ is the creation operator of an atom in $|b\rangle$ with momentum $\mathbf{k}$.

Like in an atom laser setup [15], two lasers are then used to induce two-photon Raman transitions between $|a\rangle$ and $|b\rangle$. The lasers have a two-photon Rabi frequency $\Omega$, and their frequencies and momentum differences are $\omega_{L}=$ $\omega_{1}-\omega_{2}$ and $\mathbf{k}_{L}=\mathbf{k}_{1}-\mathbf{k}_{2}$ respectively. When tuning them close to a two-photon resonance and far from single photon resonances, an effective Hamiltonian is obtained which in the interaction picture can be written as $(\hbar=1)$

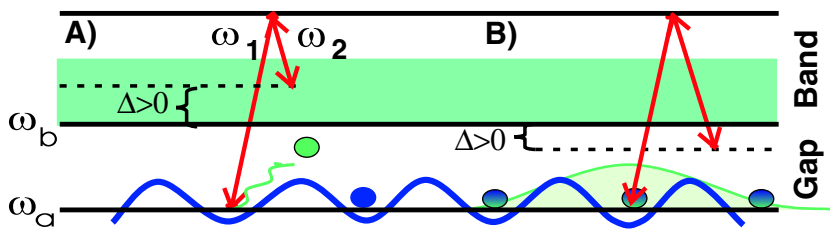

FIG. 1 (color online). Schematic diagram. Lasers produce twophoton Raman transitions to an untrapped state. A: For laser detunings $\Delta>0$, transitions are into a band of nontrapped states. B: $\Delta<0$, transitions are into the gap region, and a trappeduntrapped atom bound state is formed. 


$$
H_{\text {int }}=\sum_{\mathbf{j}} \sum_{\mathbf{k}} g_{k}\left(b_{\mathbf{k}}^{\dagger} \sigma_{\mathbf{j}} e^{i \Delta_{\mathbf{k}} t-i\left(\mathbf{k}-\mathbf{k}_{L}\right) \cdot \mathbf{r}_{\mathbf{j}}}+\text { H.c. }\right) .
$$

Here $\mathbf{r}_{\mathbf{j}}$ denotes the positions in the lattice, and $\Delta_{\mathbf{k}}=$ $k^{2} / 2 m-\Delta$, with $\Delta=\omega_{L}-\left(\omega_{b}-\omega_{a}\right)$ the laser detuning (and $\omega_{a}=\omega_{a}^{0}+\omega_{0} / 2$ ). The coupling constants are $g_{\mathbf{k}}=$ $\Omega e^{-X_{0}^{2}\left(\mathbf{k}-\mathbf{k}_{L}\right)^{2} / 2}\left(8 \pi^{3 / 2} X_{0}^{3} / V\right)^{1 / 2}$, where $X_{0}=\left(1 / m \omega_{0}\right)^{1 / 2}$ is the size of the wave function at each site.

The similarity of Hamiltonian (1) with that describing the interaction of atoms with the electromagnetic field is apparent. The dispersion relation of the atomic bath, contained in $\Delta_{k}$, resembles that of the radiation field in a three dimensional and infinite PC near the band-edge $[10,13,14]$. Furthermore, in our setup one can easily control several external parameters: $\Omega$, which determines the coupling strength, $\Delta$, which determines the resonance conditions, the number of atoms $N$ and sites $M$, the lasers wave vectors $\mathbf{k}_{L}$, and the dimension of the trap and the lattice. Thus, we expect to observe a rich variety of phenomena, ranging from some well-known from the field of Quantum Optics, to other which are difficult to access in that field. We will start out with a single excitation $(N=M=1)$, and then consider collective effects $(M>1)$, for the different regimes dictated by the control parameters $\Omega$ and $\Delta$.

An atom within a single trap constitutes the simplest setup but it still gives a very good insight into the problem. The wave function of the system has the form $|\Psi(t)\rangle=$ $A(t)|1,\{0\}\rangle+\sum_{\mathbf{k}} B_{\mathbf{k}}(t)\left|0,1_{\mathbf{k}}\right\rangle$, where $|1,\{0\}\rangle$ describes the atom in the trapped state and no free atom present, and $\left|0,1_{\mathbf{k}}\right\rangle$ represents no atom in the trapped state and a single untrapped atom in the mode $\mathbf{k}$. Choosing $\mathbf{k}_{L}=0$, and using the Schrödinger equation we have $\dot{A}(t)=$ $-\int_{0}^{t} d \tau G(t-\tau) A(\tau)$, where

$$
G(t)=\sum_{\mathbf{k}} g_{k}^{2} e^{-i \Delta_{\mathbf{k}} t}=\Omega^{2} \frac{e^{i \Delta t}}{\left(1+i \omega_{0} t / 2\right)^{3 / 2}},
$$

is the correlation function of the environment. An analytical solution can be obtained by assuming that trapped atoms are strongly confined so that $\omega_{0} \gg \Omega, \Delta$. For a $3 D$ bosonic field, this leads to $G_{\infty}(t)=-\alpha e^{i(\Delta t+\pi / 4)} / t^{3 / 2}$, which is singular at the origin, but describes correctly times $t \gg 1 / \omega_{0}$. Except for the value of $\alpha=$ $\left(2 / \omega_{0}\right)^{3 / 2} \Omega^{2}, G_{\infty}(t)$ is identical to the correlation function of the radiation field within an anisotropic PC [10,13,14]. In the same way, a $1 \mathrm{D}$ environment (produced by trapping $|b\rangle$ in a 1D harmonic trap), produces a correlation function similar to the one of the radiation field in isotropic PCs. From the Laplace transform method, we get [9]

$$
A(t)=c_{1} e^{i\left(r_{1}^{2}+\Delta\right) t}+I(\alpha, \Delta, t),
$$

with $I(\alpha, \Delta, t)=\left(\alpha e^{i \pi / 4} / \pi\right) \int_{0}^{\infty} d x \frac{\sqrt{x} e^{(-x+i \Delta) t}}{(-x+i \Delta)^{2}+i \alpha^{2} x}$. Defining $r_{ \pm}=-(\alpha / 2) \pm \sqrt{(\alpha / 2)^{2}-\Delta}$ we have: (i) If $\alpha^{2} / 2>\Delta>$ 0 , then $c_{1}=0$. (ii) If $\Delta>\alpha^{2} / 2$, then $r_{1}=r_{-}$and $c_{1}=$ $\frac{2 r_{-}}{r_{-}-r_{+}}$. (iii) If $\Delta<0, r_{1}=r_{+}$and $c_{1}=\frac{2 r_{+}}{r_{+}-r_{-}}$. Note, that for $\Delta>0$ there is no trapped atom left in steady state, whereas for $\Delta<0$ this is not the case (Fig. 2). Thus, there is a quantum phase transition at $\Delta=0$ analog to that found in the spin-boson model [16]. Moreover, for $\Delta<0$, the emitted atomic field is in the form of evanescent modes exponentially localized around the trapped atom, what leads to the trapped atom-untrapped atom bound state. This follows from the probability of finding a radiated particle at position $\mathbf{r}$ at a long time $t,\left|\Psi_{b}(\mathbf{r}, t)\right|^{2}=$ $\left(c_{1} \Omega m X_{0}^{3} / 2 \pi r\right)^{2} e^{-\operatorname{Im}\left[k_{0}^{e}\right] r} e^{-\operatorname{Im}\left[r_{1}^{2}\right] t}, \quad$ where $\quad k_{0}^{e}=$ $\sqrt{2 m\left(\Delta-r_{1}^{2}\right)}$ is imaginary if $\Delta<0$. For $\Delta>0$ we have two different regimes: for $\Delta \gg \alpha^{2}$ we just have an exponential rate $\Gamma_{0}=4 \Omega^{2} \sqrt{2 \pi \Delta / \omega_{0}^{3}}$, whereas in the opposite limit the evolution does not follow such a law. The first corresponds to the Markovian regime, where the correlation time $\tau_{c} \simeq \Delta^{-1}$ of the environment (here untrapped atoms) is shorter than the typical evolution time of the trapped atom $\left(\Gamma_{0}^{-1}\right)$. For finite $\omega_{0}, \Gamma_{0}=\int_{0}^{\infty} d t G(t)=$ $4 \Omega^{2}\left(-i / \omega_{0}+\sqrt{2 \pi \Delta / \omega_{0}^{3}} e^{-2 \Delta / \omega_{0}}\left(1+\operatorname{Erf}\left[i \sqrt{2 \Delta / \omega_{0}}\right]\right)\right)$, where the first term is the analogue to the Lamb shift. In this case, the structure of $A(t)$ is similar to (3), but the phase transition above described is shifted to $\Delta=4 \Omega^{2} / \omega_{0}$ (see inset Fig. 2).

We now study the dynamics of atoms in a lattice with $M$ sites, choosing $\omega_{0} \gg \Omega, \Delta$. Guided by the previous analysis, we will consider the regimes $\Delta>0$ (Markovian and non-Markovian), as well as $\Delta<0$.

In the limit where $\Gamma_{\text {coll }} \tau_{c} \ll 1$, where $\Gamma_{\text {coll }}$ gives the typical evolution time of the trapped atoms, we can analyze the problem under the Born-Markov approximation. The dynamics of the atoms in the lattice is dictated by the quantities

$$
\Gamma_{|\mathbf{i}-\mathbf{j}|}=\int_{0}^{\infty} d \tau G_{\mathbf{i}-\mathbf{j}}(\tau)=i\left|\Gamma_{0}\right| \xi \frac{e^{-\nu|\mathbf{i}-\mathbf{j}| / \xi+i \mathbf{r}_{\mathbf{i}-\mathbf{j}} \cdot \mathbf{k}_{L}}}{|\mathbf{i}-\mathbf{j}|}
$$

for $\mathbf{i} \neq \mathbf{j}$. Here, $\nu=i, 1$ for $\Delta>0$ and $\Delta<0$, respec-

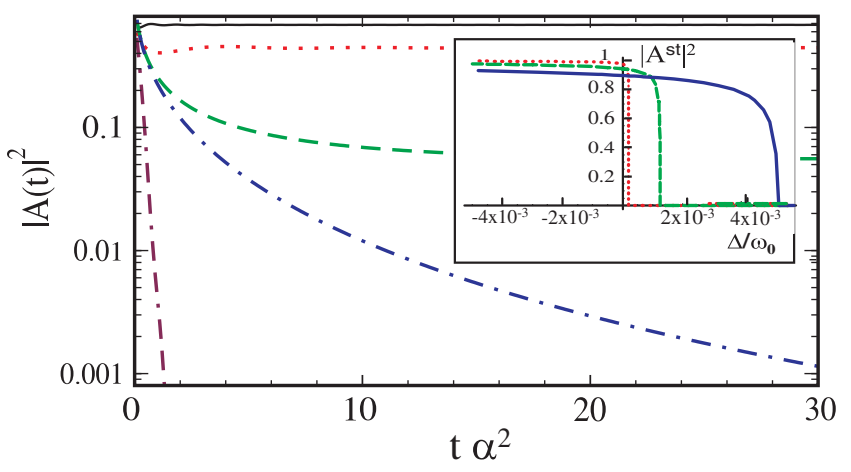

FIG. 2 (color online). Evolution of $|A(t)|^{2}$ from Eq. (3) in logarithmic scale for $\Delta / \alpha^{2}=-8,-1,-0.2,0.2,8\left(\omega_{0}=\infty\right)$, corresponding to solid, dotted, dashed, dot-dashed, and dot-dotdashed lines, respectively. One can recognize the regimes explained in the text. Inset: Steady state population $\left|A^{\text {st }}\right|^{2}=$ $|A(\infty)|^{2}$. Solid, dashed, and dotted lines correspond, respectively, to $\Omega / \omega_{0}=0.025,0.01,0.005$. 
tively, and the correlation function $G_{\mathbf{i}-\mathbf{j}}(t)=$ $\sum_{\mathbf{k}} g_{k}^{2} e^{i \mathbf{r}_{\mathbf{i}-\mathbf{j}} \cdot\left(\mathbf{k}-\mathbf{k}_{L}\right)-i \Delta_{k} t}$ is now

$$
G_{\mathbf{i}-\mathbf{j}}(t)=G(t) e^{-r_{\mathbf{i}-\mathbf{j}}^{2} /\left(4 X_{0}^{2} \nu_{t}^{2}\right)} e^{i \mathbf{r}_{\mathbf{i}-\mathbf{j}} \cdot \mathbf{k}_{L}}
$$

with $G(t)$ given by (2). Similar to the radiative case, the coefficients $\Gamma_{|\mathbf{i}-\mathbf{j}|}$ describe the dipolar interactions between the sites $\mathbf{i}$ and $\mathbf{j}$. The quantity $\xi=1 /\left(\left|k_{0}\right| d_{0}\right)$, with $k_{0}=\sqrt{2 m \Delta}$ [17], quantifies the range of the interactions which, according to (4), has a Yukawa form for the case $\Delta<0$.

For $\Delta>0$, the situation under study resembles that of a set of $M$ atoms in a lattice interacting with the electromagnetic field in a $\Lambda$-scheme as described, e.g., in [18], with $k_{0}$ corresponding to the resonant wave number, and $N$ to the number of excited atoms. However, here $\mathbf{k}_{L}$ is a difference between wave-vectors, and hence it can be varied by changing the relative directions of the lasers. Following the light-matter analogy, collective phenomena like multiple scattering or superradiance are expected. These are determined by $\xi$ and the analogous of the optical depth, which for a cubic lattice is $\chi=\pi^{3 / 2} M^{1 / 3} \xi^{2}$.

We now consider one atom symmetrically distributed along $M$ sites, a situation that has been analyzed in Ref. [18] for the light-matter interaction case. This analysis can be translated straightforwardly to our system, when the lasers are tuned to fulfill the phase matching condition, $\left|\mathbf{k}_{L}\right|=\left|k_{0}\right|$. Hence, two interesting regimes where collective effects are present can be obtained [18]: First, choosing $\xi \pi>1$, atoms in $|b\rangle$ are emitted in the direction of $\mathbf{k}_{L}$ with a collective rate $\Gamma_{\text {coll }} \sim \chi \Gamma_{0}$. Second, by choosing $\xi>M^{2 / 3}$ atoms are emitted with a rate $\Gamma_{\text {coll }}=M \Gamma_{0}$ characteristic of Dicke superradiance [11].

Let us now study $N$ atoms within $M$ sites, considering the limit where $\xi>1$, where according to (4) collective effects are expected in the dynamics. From now on, we take laser directions such that $\left|\mathbf{k}_{L}\right| d_{0} M^{1 / 3} \ll 1$, so that the momentum of the laser plays no role in the dynamics. Hence, we get

$$
\begin{aligned}
\frac{d\left\langle\sigma_{\mathbf{i}}^{3}\right\rangle}{d t} & =-4 \operatorname{Re}\left[\sum_{\mathbf{j}} \Gamma_{|\mathbf{j}-\mathbf{i}|}\left\langle\sigma_{\mathbf{i}}^{+} \sigma_{\mathbf{j}}\right\rangle\right] \frac{d\left\langle\sigma_{\mathbf{i}}^{+} \sigma_{\mathbf{j}}\right\rangle}{d t} \\
& =\sum_{l} \Gamma_{|\mathbf{I}-\mathbf{i}|}^{*}\left\langle\sigma_{l}^{+} \sigma_{\mathbf{i}}^{3} \sigma_{\mathbf{j}}\right\rangle+\Gamma_{|\mathbf{I}-\mathbf{j}|}\left\langle\sigma_{\mathbf{i}}^{+} \sigma_{\mathbf{j}}^{3} \sigma_{l}\right\rangle,
\end{aligned}
$$

with rates given by (4). Here, $\sigma_{\mathbf{i}}^{3}=2 \sigma_{\mathbf{i}}^{+} \sigma_{\mathbf{i}}-1$, and all the operators are evaluated a time $t$. Let us first analyze the atomic emission that occurs for positive detuning $\Delta>0$. We focus on the rate of emission of atoms in all directions, which is given by $\mathcal{R}(t) \approx-\sum_{\mathbf{j}} d\left\langle\sigma_{\mathbf{j}}^{3}\right\rangle / d t$, for different values of $\xi$. If sites evolve independently, $\mathcal{R}(t)$ decays exponentially. However, when $\xi>1$ and collective effects are present, $\mathcal{R}(t)$ does no longer decay exponentially and, furthermore, it presents positive slopes at initial times. This is shown in Fig. 3 for a $1 D$ lattice, where it is observed that collective effects occur for $\xi>1$. This result is obtained with (6) by using the semiclassical decoupling $\left\langle\sigma_{l}^{+} \sigma_{\mathbf{i}}^{3} \sigma_{\mathbf{j}}\right\rangle=\left\langle\sigma_{\mathbf{i}}^{3}\right\rangle\left\langle\sigma_{l}^{+} \sigma_{\mathbf{j}}\right\rangle$, that is based on neglecting atomic quantum fluctuations [12]. Nevertheless, the change of sign in the slope can be obtained analytically by differentiating Eq. (6) at $t=0$ without the use of any approximation.

For negative detunings, $\Delta<0$, the rates (4) are purely imaginary, and the system has an effective Hamiltonian

$$
H_{\mathrm{eff}}^{\Delta<0}=\sum_{\mathbf{i}, \mathbf{j}} J_{|\mathbf{i}-\mathbf{j}|} \sigma_{\mathbf{i}}^{\dagger} \sigma_{\mathbf{j}},
$$

where $J_{|\mathbf{i}-\mathbf{j}|}=i \Gamma_{|\mathbf{i}-\mathbf{j}|}$ is a real and negative quantity, so that (7) is Hermitian and describes a coherent spin-spin interaction of ferromagnetic type. This interaction may have interesting applications in the field of quantum simulation. Furthermore, for $\xi \gg 1$ it gives a Coulomb-like interaction very difficult to obtain with other techniques.

Let us now study the non-Markovian limit, where the system also becomes strongly interacting. We consider that all the atoms are initially in the lattice (i.e., $N=M$ ), and the limit $M \gg 1$. Using the mean field or Hartree approximation [19], the evolution of $y(t)=\sum_{\mathbf{j}}\left\langle\sigma_{\mathbf{j}}(t)\right\rangle / M$ and $z(t)=\sum_{\mathbf{j}}\left\langle\sigma_{\mathbf{j}}^{3}(t)\right\rangle / M$ can be written as

$$
\begin{aligned}
& \frac{d y(t)}{d t}=M \int_{0}^{t} d \tau G_{\mathrm{coll}}(t-\tau) y(\tau) z(t) \\
& \frac{d z(t)}{d t}=-4 M \operatorname{Re}\left[\int_{0}^{t} d \tau G_{\mathrm{coll}}(t-\tau) y^{*}(\tau) y(t)\right] .
\end{aligned}
$$

Here, $G_{\text {coll }}(t)=(1 / M) \sum_{\mathbf{n}} G_{\mathbf{n}}(t)$, with $G_{\mathbf{n}}(t)$ defined in (5). Because of the non-Markovian structure of the equations, the mean field approximation considered predicts that in the steady state $y^{\text {st }} \neq 0$. This is shown in Fig. 3 for $\Delta=0$, where we have taken an initial infinitesimal value $y(0)=$ $10^{-6}[20]$, and $z(0)=1$. This is analogous to the sponta-

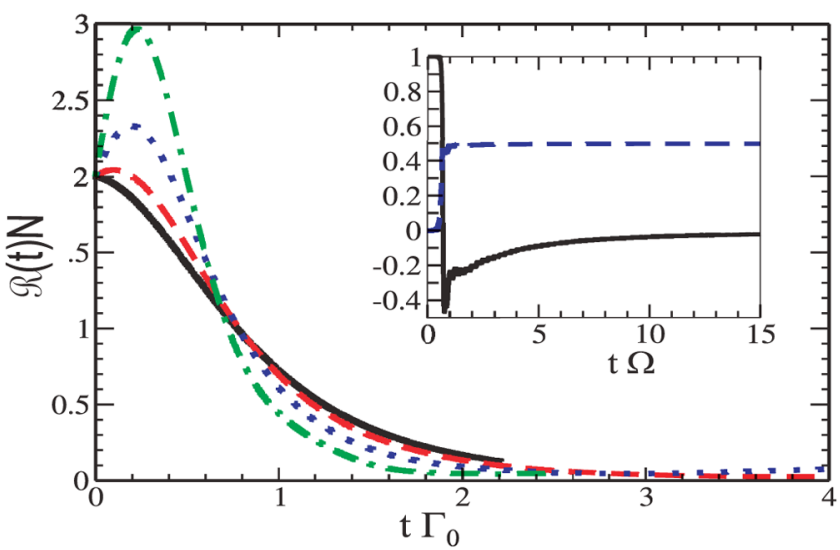

FIG. 3 (color online). Rate of atomic emission from a $1 D$ lattice with $N=M=100$ atoms. Solid, dashed, dotted and dot-dashed curves correspond to $\xi=0.9,1.25,2,3.33$, respectively. Inset: Spontaneous symmetry breaking and nonzero steady state population. Solid and dashed curves represent $z(t)$ and $y(t)$ respectively, evolving according to (8), for $N=M=$ $10^{3}$ atoms, $\Delta=0$, and $\omega_{0}=50$. 
neous polarization of the system described in [10] for atoms in PCs, and similar to the spontaneous symmetry breaking predicted by the semiclassical theory of the laser (see [19] and references). Figure 3 also shows that the nonMarkovian effects lead to a nonzero steady state population, i.e., $z^{\text {st }} \neq-1$.

The phenomena here described may be observed with state of the art experimental setups using state-dependent potentials [1] in the Mott insulator regime [2] for the lattice atoms and choosing $\Omega \ll \omega_{0}$ to avoid the occupation of other bands. The gravity force acting on the untrapped atoms $|b\rangle$ has not been considered, and it can be compensated in an experiment with a magnetic field gradient [21]. This gradient may be combined with bias field to tune the scattering properties such that the on-site interaction of atoms in $|a\rangle, U_{a a}>0$, while the interaction of atoms in $|b\rangle$ and in different states are $U_{b b}, U_{a b} \sim 0$, respectively (see [4] and references).

In order to prepare the system in the simplest regime $(N=M=1)$, note that it is not required to have only a single atom in the whole lattice, as long as the atoms do not interact with each others (i.e., $\xi \ll 1$ and initially localized). Then, one could easily monitor the decay as a function of time by simply measuring how many atoms remain in $|a\rangle$. The presence of the bound state of the untrapped atoms should be visible in the momentum distribution after free expansion. By preparing a few atoms each of them coherently distributed among $M$ sites in disjoint regions, several copies of the setup consisting in $N=1$ atom within $M$ sites could be realized. For an initial Mott insulator state $(N=M)$ it should also be possible to observe superradiant effects by looking at the slope of the decay rate for short times, and for $\Delta<0$ the nearestneighbor interaction induced via virtual transitions to the untrapped state, as follows from Eq. (7). Other properties of the system, like the directionality in the emission, could be characterized by measuring the emitted atoms. All those phenomena require $\xi \geq 1$, i.e. $|\Delta| \leq 1 /\left(2 m d_{0}^{2}\right)$, as well as the Markovian limit, $\Gamma_{\text {coll }} \leq|\Delta|$. Observing superradiance for long times (i.e., the whole shape of Fig. 3), as well as coherent interactions in Eq. (7) beyond nearest neighbors, may be limited by decoherence effects caused by random magnetic fields which shift $|a\rangle$ and $|b\rangle$ differently. A possible way around this is to use lighter atomic species, like $\mathrm{Na}$ and $\mathrm{Li}$, where those conditions are relaxed. For instance, the time scale of Fig. 3 is of the order of a few tens of milliseconds for $\mathrm{Rb}$, but of the order of only a few milliseconds for Li. The collective non-Markovian effects should be easy to observe by choosing $\Delta \simeq 0$.

The proposed setup may be advantageous to observe some phenomena with respect to atoms interacting with light in a PC. First, an atom in a PC has a fixed transition frequency and its detuning with respect to the band-gap edge determines the system dynamics. Here, the equivalent quantity is $\Delta$, which together with other laser parameters can be freely varied, allowing to describe a richer variety of regimes. Second, the detection techniques developed for atoms in optical lattices [22] may allow to measure features, like the analogue to the photon-atom bound state, that are difficult to measure in PCs. Also, other interesting phenomena could be explored with this set-up. For example, for $\chi \gg 1$ light-matter interface schemes can be used to control the emission direction of the atoms, or to map the state of trapped atoms into that of untrapped atoms [23]. Besides, if $|b\rangle$ is affected by a trap that is wider than that of $|a\rangle$, other types of interactions, like the one described by the Jaynes Cummings or the so-called Tavis Cummings model, can be implemented. Additionally, this system may be tuned to explore other regimes which cannot be reached in quantum optics. For example, the ones in which the initial state of the atoms in the lattice is a superfluid, or a Tonks gas. Finally, by choosing $U_{a b} \neq 0$ new dynamics could be described, in which the scattering between atoms in $|a\rangle$ and $|b\rangle$ compete with collective decay.

We thank M. Aguado, M. C. Bañuls, I. Bloch, S. Dürr and G. Giedke for fruitful discussions. Work supported by EU projects (SCALA, COMPASS), DFG Munich-Centre for Advanced Photonics, and Spanish MEC.

*Ines.devega@mpq.mpg.de

[1] D. Jaksch et al., Phys. Rev. Lett. 81, 3108 (1998); O. Mandel et al., Nature (London) 425, 937 (2003).

[2] M. Greiner et al., Nature (London) 415, 39 (2002); S. Fölling et al., Nature (London) 434, 481 (2005).

[3] B. Paredes et al., Nature (London) 429, 277 (2004).

[4] I. Bloch et al., Rev. Mod. Phys. 80, 885 (2008).

[5] A. Recati et al., Phys. Rev. Lett. 94, 040404 (2005).

[6] Peter P. Orth et al., Phys. Rev. A 77, 051601(R) (2008).

[7] K. Molmer, Phys. Rev. Lett. 90, 110403 (2003).

[8] S. John, Phys. Rev. Lett. 58, 2486 (1987); E. Yablonovitch, Phys. Rev. Lett. 58, 2059 (1987).

[9] M. Woldeyohannes and S. John, J. Opt. B 5, R43 (2003).

[10] S. John and T. Quang, Phys. Rev. Lett. 74, 3419 (1995).

[11] R. H. Dicke, Phys. Rev. 93, 99 (1954).

[12] A. V. Andreev et al., Cooperative effects in optics, superradiance, and phase transitions (Institute of Physics Publishing, Bristol and Philadelphia, 1993).

[13] S. John and T. Quang, Phys. Rev. A 50, 1764 (1994).

[14] I. de Vega et al., Phys. Rev. A 71, 023812 (2005); I. de Vega et al., Phys. Rev. A 77, 043836 (2008).

[15] G. M. Moy and C. M. Savage, Phys. Rev. A 56, R1087 (1997).

[16] A. J. Leggett et al., Rev. Mod. Phys. 59, 1 (1987).

[17] Note that $k_{0}^{e} \approx k_{0}$, within the weak coupling limit.

[18] D. Porras and J. I. Cirac, Phys. Rev. A 78, 053816 (2008).

[19] H.P. Breuer and F. Petruccione, The Theory of Open Quantum Systems (Oxford Univ. Press, New York, 2002).

[20] However, note that the steady state does not depend on the choice of $y(0)$.

[21] T. Weber et al., Science 299, 232 (2003).

[22] S. Fölling et al., Nature (London) 434, 481 (2005).

[23] K. Hammerer et al. Phys. Rev. A 70, 044304 (2004). 